\title{
Superconductivity of the Two-Dimensional Penson-Kolb Model
}

\author{
W.R. Czart and S. Robaszkiewicz \\ Institute of Physics, Adam Mickiewicz University \\ Umultowska 85, 61-614 Poznań
}

(Received July 2, 2001)

\begin{abstract}
Two-dimensional $(d=2)$ Penson-Kolb model, i.e. the tight-binding model with the pair-hopping (intersite charge exchange) interaction, is considered and the effects of phase fluctuations on the $s$-wave superconductivity of this system are discussed within the Kosterlitz-Thouless scenario. The London penetration depth $\lambda$ at $T=0$, the Kosterlitz-Thouless critical temperature $T_{c}$, and the Hartree-Fock approximation critical temperature $T_{\mathrm{p}}$ are determined as a function of particle concentration and interaction. The Uemura type plots ( $T_{\mathrm{c}}$ vs. $\lambda^{-2}(0)$ ) are derived. Beyond weak coupling and for low concentrations they show the existence of universal scaling: $T_{\mathrm{c}} \sim 1 / \lambda^{2}(0)$, as it was previously found for the attractive Hubbard model and for the models with intersite electron pairing.
\end{abstract}

PACS numbers: 74.20.-z, 71.28.+d, 74.25.Ha

\section{Introduction}

The Penson-Kolb (PK) model is one of the conceptually simplest models for studying superconductivity of the narrow band systems with short coherence length [1-8]. The model Hamiltonian has the form

$$
\begin{aligned}
H= & -t \sum_{\langle i j\rangle \sigma}\left(\mathrm{e}^{\mathrm{i} \Phi_{i j}} c_{i \sigma}^{+} c_{j \sigma}+\text { h.c. }\right)-\sum_{i \sigma} \mu n_{i \sigma} \\
& -\frac{1}{2} J \sum_{\langle i j\rangle}\left(\mathrm{e}^{2 \mathrm{i} \Phi_{i j}} c_{i \uparrow}^{+} c_{i \downarrow}^{+} c_{j \downarrow} c_{j \uparrow}+\text { h.c. }\right),
\end{aligned}
$$

where $n_{i \sigma}=c_{i \sigma}^{+} c_{i \sigma}, t$ is the single electron hopping integral, $J$ is the pair-hopping (intersite charge exchange) interaction, $\mu$ is the chemical potential, the limit $\langle i j\rangle$ 
restricts the sum to nearest neighbors (nn). The Peierls factors in Eq. (1) account for the coupling of electrons to the magnetic field via its vector potential $\boldsymbol{A}(\boldsymbol{r})$ : $\Phi_{i j}=-\frac{e}{\hbar c} \int_{R_{i}}^{R_{j}} \mathrm{~d} \boldsymbol{r} \boldsymbol{A}(\boldsymbol{r})$, and $e$ is the electron charge.

The model includes a nonlocal pairing mechanism (the pair-hopping term $J$ ) which is distinct from the on-site interaction in the attractive Hubbard (AH) model and which is the driving force of pair formation and also of their condensation. Thus, the superconducting properties and the evolution from the Cooper pair regime to the strong coupling local pair regime can be essentially different in these two models [2]. Till now the PK model has been investigated only in a few particular limits [1-8]. The main efforts concerned the ground state properties of the model in one dimension $(d=1)$ at half filling $(n=1)[3-7]$. For higher-dimensional lattices $(1<d \leq \infty)$ and arbitrary electron concentration $(0<n<2)$, where $n=(1 / N) \sum_{i \sigma}\left\langle n_{i \sigma}\right\rangle$, the model has been analysed only at $T=0$ in Refs. [2, 8] within broken symmetry Hartree-Fock approximation (HFA).

The ground state phase diagram of the half-filled $d=1$ PK model derived within the HFA [2] is in agreement with that obtained by the density matrix renormalization group method [3-5], exact Lanczos diagonalizations [6], as well as with the continuum limit field theory approaches [7]. For $J>0$ all these approaches predict a continuous second-order transition to $s$-wave pairing state at $J=0^{+}$ with no additional transition for any $J>0$. The results of Refs. [2, 8] indicate that for hypercubic lattices such behavior remains unchanged in higher dimensions (including the exactly solvable case of $d=\infty$ ) and does not depend on the band filling.

In Ref. [8] we analyzed the ground state superfluid characteristics of the PK model (critical fields, coherence length, Ginzburg ratio) for $d$-dimensional hypercubic lattices: $d=2(\mathrm{SQ})$ and $d=3(\mathrm{SC})$ and showed that at $T=0$ there is a gradual crossover from the BCS-like superconductivity of extended Cooper pairs, to the superfluidity of tightly bound local pairs with increasing $J(J>0)$.

In the present paper we will extend our investigations to the case of finite temperatures. We will focus on the two-dimensional PK model with arbitrary particle concentration and discuss its superfluid characteristics and the effects of phase fluctuations, which have not been considered up to now for this model.

In the analysis we have used a linear response theory $[9,10]$ and the electromagnetic kernel has been evaluated within HFA-random phase approximation (HFA-RPA) scheme. Going beyond the HFA we will determine $T_{\mathrm{c}}$ for $d=2 \mathrm{PK}$ model on an SQ lattice taking into account the phase fluctuations within the framework of the Kosterlitz-Thouless (K-T) scenario $[11,12]$. The critical temperature $T_{\mathrm{C}}$ for the $\mathrm{K}-\mathrm{T}$ transition is obtained by calculating the superfluid stiffness $\rho_{\mathrm{s}}$ (helicity modulus) as a function of temperature and subsequent comparison with the $\mathrm{K}-\mathrm{T}$ relation between $T_{\mathrm{c}}$ and $\rho_{\mathrm{s}}$. This type of approach has been already applied for the $d=2 \mathrm{AH}$ model $[12,13]$ and also for the models with intersite electron pairing [14-16]. In the former case a correct behavior of $T_{\mathrm{c}}$ vs. $|U| / B$, being in 
agreement with available quantum Monte Carlo (QMC) data, was found [13].

\section{General formulation}

Within the framework of the broken-symmetry Hartree-Fock approximation (HFA) the free energy of the model (1) and self-consistent equations for electron thermal averages can be determined by the standard methods with either the Green function or the equation of motion approach [2].

For $\boldsymbol{A}=\mathbf{0}$ the free energy of the superconducting $(\mathrm{S})$ phase $F_{\mathrm{S}}$ is calculated to be

$$
\frac{F_{\mathrm{S}}}{N}=\mu(n-1)+\frac{4}{z} J p^{2}+J_{0} x_{\mathrm{S}}^{2}-\frac{2}{\beta N} \sum_{k} \ln \left[2 \cosh \left(\frac{\beta E_{k}}{2}\right)\right],
$$

and the superconducting order parameter $x_{\mathrm{s}}=(1 / N) \sum_{i}\left\langle c_{i \downarrow} c_{i \uparrow}\right\rangle$, the Fock term $p=(1 / 4 N) \sum_{k \sigma} \gamma_{k}\left\langle c_{k \sigma}^{+} c_{k \sigma}\right\rangle$ and $\mu$ are determined by the equations

$$
\begin{aligned}
& \Delta=\frac{z J}{N} \sum_{k} \frac{\Delta}{2 E_{k}} \tanh \left(\frac{\beta E_{k}}{2}\right), \quad p=\frac{1}{N} \sum_{k} \bar{\epsilon}_{k} \gamma_{k} \frac{1}{2 E_{k}} \tanh \left(\frac{\beta E_{k}}{2}\right), \\
& n-1=-\frac{2}{N} \sum_{k} \bar{\epsilon}_{k} \frac{1}{2 E_{k}} \tanh \left(\frac{\beta E_{k}}{2}\right),
\end{aligned}
$$

where $\bar{\epsilon}_{k}=\epsilon_{k}-\mu, E_{k}=\sqrt{\bar{\epsilon}_{k}^{2}+\Delta^{2}}, \Delta=J_{0} x_{\mathrm{s}}, J_{0}=z J, \epsilon_{k}=-\tilde{t} \gamma_{k}, \tilde{t}=$ $t+2 p J / z, \gamma_{k}=2 \sum_{\alpha} \cos k_{\alpha}, \alpha=x, y, \ldots, z$ is the number of nearest neighbors, $\beta=1 / k_{\mathrm{B}} T$. For SQ lattice $z=4$ and the electronic band width $B=2 z t=8 t$.

From the linear response theory $[9,10]$ the expectation value of the Fourier transform of the total current operator for the Hamiltonian (1) is

$$
J_{\alpha}(\boldsymbol{q}, \omega)=N \frac{c}{4 \pi} \sum_{\alpha^{\prime}}\left[\delta_{\alpha \alpha^{\prime}} K_{\alpha}^{\text {dia }}+K_{\alpha \alpha^{\prime}}^{\text {para }}(\boldsymbol{q}, \omega)\right] A_{\alpha^{\prime}}(\boldsymbol{q}, \omega)
$$

The diamagnetic part of kernel evaluated within HFA-RPA scheme has the form

$$
K_{\alpha}^{\text {dia }}=\frac{8 \pi e^{2}|t|}{\hbar^{2} c^{2} a} \frac{1}{N} \sum_{k}\left[1-\frac{\bar{\epsilon}_{k}}{E_{k}} \tanh \left(\frac{\beta E_{k}}{2}\right)\right] \cos \left(k_{\alpha}\right)-\frac{32 \pi e^{2}}{\hbar^{2} c^{2} a} \frac{J_{0}}{z} x_{\mathrm{s}}^{2} .
$$

In the static limit and for $q \rightarrow 0$ for the transverse part of the paramagnetic kernel we obtain

$$
K_{x x}^{\text {para }}(\omega=0)=\frac{8 \pi e^{2} t^{2}}{\hbar^{2} c^{2} a} \frac{1}{N} \sum_{k} \frac{\sin ^{2} k_{x}}{k_{\mathrm{B}} T \cosh ^{2}\left(\frac{\beta E_{k}}{2}\right)} .
$$

In the local approximation (London limit) the magnetic penetration depth is determined in terms of the transverse part of the total kernel as

$$
\lambda=\left[-K_{x}^{\text {dia }}-K_{x x}^{\text {para }}(\omega=0)\right]^{-1 / 2} .
$$

From Eqs. (3) one can calculate the HFA transition temperature $T_{\mathrm{p}}$ at which the gap amplitude vanishes $(\Delta \rightarrow 0)$ and which gives the estimation of the pair-formation temperature. 
Except of the case of infinite-dimensional lattice $(d \rightarrow \infty)$ the superconducting phase transition will occur at the critical temperature $T_{c}$ being lower than $T_{\mathrm{p}}$ due to the phase fluctuation effects. For $d=2$ lattice the $T_{\mathrm{c}}$ can be derived within the Kosterlitz-Thouless theory [11, 12], which describes the transition in terms of vortex pair unbinding transition, using the $\mathrm{K}-\mathrm{T}$ relation for the universal jump of the superfluid stiffness at $T_{c}$ :

$$
\frac{2}{\pi} k_{\mathrm{B}} T_{\mathrm{c}}=\rho_{\mathrm{s}}\left(T_{\mathrm{c}}\right)
$$

where $\rho_{\mathrm{s}}$ is the superfluid stiffness (helicity modulus), being directly related to the London penetration depth (Eq. (7)):

$$
\rho_{\mathrm{s}}(T)=\frac{\hbar^{2} c^{2}}{16 \pi e^{2}} \lambda^{-2}=\frac{-\hbar^{2} c^{2}}{16 \pi e^{2}}\left[K_{x}^{\text {dia }}+K_{x x}^{\text {para }}(\omega=0)\right]
$$

\section{Results}

The asymptotic forms of the London penetration depth at $T=0$ derived in the weak $\left(J_{0} / B \ll 1\right)$ and strong $\left(J_{0} / B \gg 1\right)$ coupling limits are given in Appendix A (Eqs. (A2), (A4), (A6), (A8)), whereas the numerical plots of this quantity as a function of $J_{0} / B$ and as a function of $n$ are presented in Figs. 1 and 2, respectively. For a comparison, in Fig. 1 the corresponding plots $\left(1 / \lambda^{2}\right.$ vs. $|U| / B)$ calculated for the AH model [10a] are also shown by dashed lines.

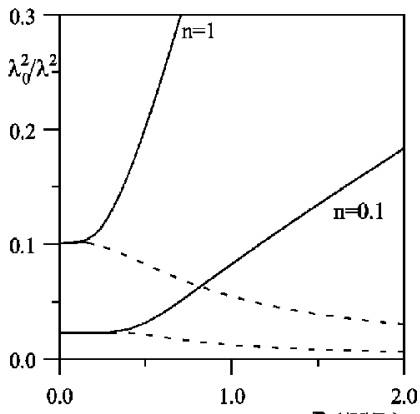

Fig. 1

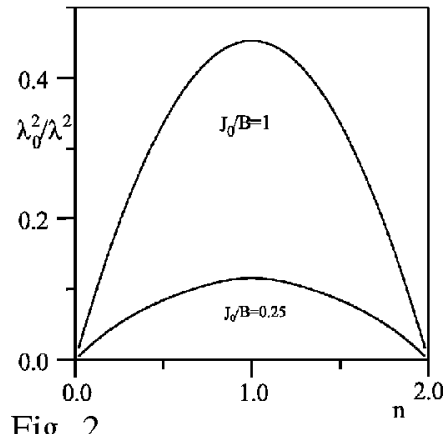

Fig. 2

Fig. 1. The inverse square penetration depth $1 / \lambda^{2}$ at $T=0$ as a function of $J_{0} / B$ for $n=1$ and $n=0.1$. Dashed lines show the corresponding plots for the AH model. $\lambda_{0}=\frac{\hbar c}{e} \sqrt{\frac{1}{4 \pi B}}, B=8 t$.

Fig. 2. Concentration dependence of $1 / \lambda^{2}$ at $T=0$ for two different values of $J_{0} / B, J_{0} / B=0.25$ and $J_{0} / B=1.0$.

Let us notice the increase in $\lambda^{-2}$ as a function of $J$ (cf. Fig. 1), i.e. the behavior being qualitatively different than that found for the $\mathrm{AH}$ model, where $\lambda^{-2}$ continuously decreases with increasing $|U| / B$ (cf. Fig. 1 and Ref. [10]). 
In Fig. 3 the plots of $T_{\mathrm{p}}$ and $T_{\mathrm{c}}$ versus $n$ for fixed values of $J_{0} / B$ are presented, whereas the evolution of the transition temperatures with increasing $J_{0} / B$ for fixed $n$ is shown in Fig. 4. Except of the weak coupling regime $J / t \ll 1$ there is a strong influence of the phase fluctuations on the superconducting pairing. The $\mathrm{K}-\mathrm{T}$ transition temperature $T_{\mathrm{c}}$ can be much lower than $T_{\mathrm{p}}$ and the highest reduction is observed at small electron concentrations. The expressions for $T_{\mathrm{p}}$ and $T_{\mathrm{C}}$ derived in the asymptotic limits of weak and strong coupling are summarized in Appendix B (Eqs. (B2)-(B9)).
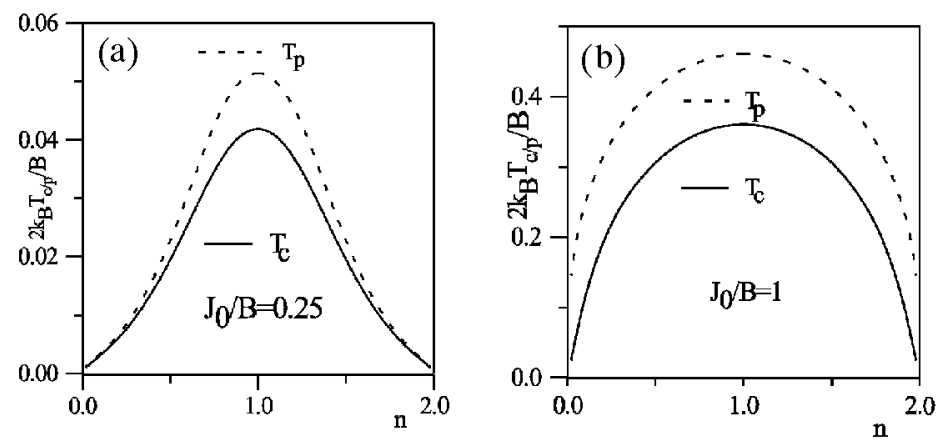

Fig. 3. Transition temperatures versus $n$ for two fixed values of $J_{0} / B$ : (a) 0.25 , (b) 1 . Solid and dashed lines denote $T_{\mathrm{c}}$ and $T_{\mathrm{p}}$ critical temperatures, respectively.

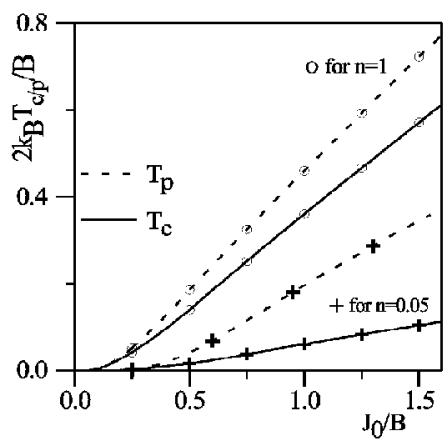

Fig. 4. Transition temperatures versus $J_{0} / B$ for $n=1$ (curves with circles) and $n=0.05$ (curves with crosses). Denotations as in Fig. 3

With decreasing temperature, at $T_{\mathrm{p}}$ the gap in quasiparticle energy spectrum opens up, but there is no real phase transition to a superconducting state yet. Only at $T_{c}$, the phase coherence sets in and the transition to a phase with bound vortex-antivortex pairs occurs. Thus in the region between $T_{\mathrm{p}}$ and $T_{\mathrm{c}}$ one has a phase of incoherent $s$-wave pairs. $T_{\mathrm{p}}$ appears to be a rather reliable estimate for the pair formation temperature and the appearance of the pseudogap $[13,15,16]$. 


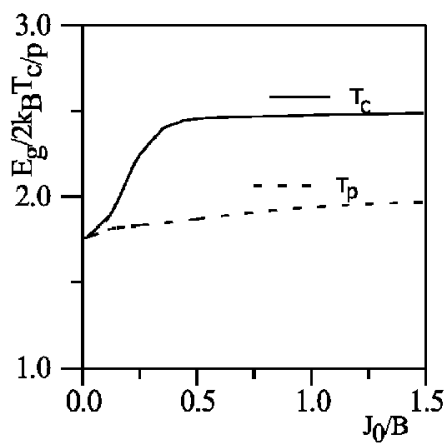

Fig. 5. The gap to critical temperature ratio $E_{\mathrm{g}}(0) / 2 k_{\mathrm{B}} T_{\mathrm{c} / \mathrm{p}}$ plotted as a function of $J_{0} / B$ for $n=1$. Solid and dashed lines correspond to $T_{\mathrm{c}}$ and $T_{\mathrm{p}}$, respectively.

However, it is by no means a rigorous border line, since some pairing correlations are present at all temperatures.

The phase fluctuations enhance the ratio of the gap $\left(E_{\mathrm{g}}=\min \left(E_{k}\right)-\right.$ $\left.\max \left(-E_{k}\right)\right)$ at $T=0$ to the critical temperature in comparison to the BCS value. The ratio $E_{\mathrm{g}}(0) / 2 k_{\mathrm{B}} T_{\mathrm{c} / \mathrm{p}}$ evaluated as a function of $J_{0} / B$ for $n=1$ is shown in Fig. 5. Notice that this ratio can be substantially higher for $T_{\mathrm{c}}$ than for $T_{\mathrm{p}}$. As we found, the enhancement of the ratio is strongest at low concentrations even by a factor 10 (cf. also Eqs. (B6), (B9) in Appendix B). Only for $J / t \rightarrow 0$ we recover the BCS ratio for $s$-wave pairing: $E_{\mathrm{g}}(0) / 2 k_{\mathrm{B}} T_{\mathrm{c}} \approx 1.76$.

In Fig. 6 we have shown the Uemura type plots $T_{\mathrm{c}}$ vs. $1 / \lambda^{2}[17,18]$ for the $d=2$ PK model, calculated for several fixed values of $J_{0} / B$ with the controling

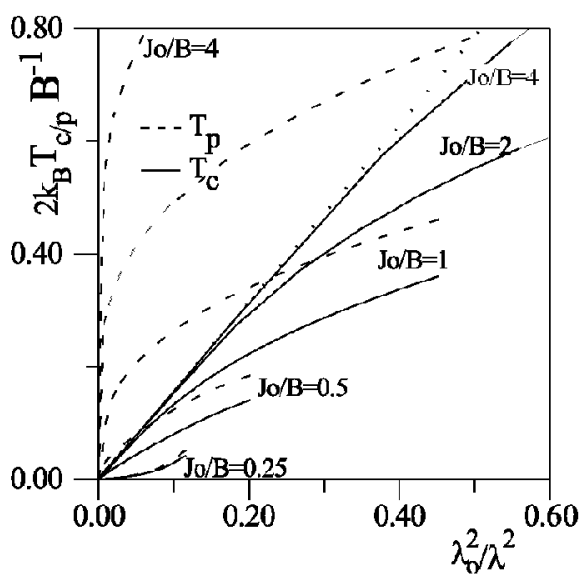

Fig. 6. The Uemura-type plots: $2 k_{\mathrm{B}} T_{\mathrm{c}} / B$ vs. $\lambda_{0}^{2} / \lambda^{2}$ with the controling variable $n$, derived for several fixed values of $J_{0} / B$. SQ lattice, $\lambda_{0}^{2} / \lambda^{2}=4 \rho_{\mathrm{s}}(0) / B$. Dashed lines denote the corresponding plots with $T_{\mathrm{c}}$ replaced by $T_{\mathrm{p}}$. The straight dotted line gives an upper bound for the phase ordering temperature $\pi \rho_{s}(0) / 2$. 
variable $n$. The straight dotted line $\pi \rho_{\mathrm{s}}(0) / 2$ gives an upper bound for the phase ordering temperature. Except for weak coupling limit the $T_{c}$ vs. $1 / \lambda^{2}$ curves have a shape similar to the experimental Uemura plots [17] and for small $n$ the points follow the universal $\pi \rho_{\mathrm{s}}(0) / 2$ line. Also, we see that analogous plots with $T_{\mathrm{p}}$ cannot account for the scaling (Fig. 6, dashed lines).

\section{Conclusions}

We have studied here the superconducting properties of the two-dimensional PK model for arbitrary electron density and coupling strength $J>0$, taking into account the effects of phase fluctuations within the framework of $\mathrm{K}-\mathrm{T}$ scenario. Our results indicate a possibility of a new disordered phase, between $T_{\mathrm{p}}$ and $T_{\mathrm{c}}$, in which pairs are incoherent, but the single-electron excitation spectrum has a gap. Transition to the superconducting state takes place at $T_{\mathrm{c}}$, at which the phase coherence sets in.

We have shown that in the $d=2 \mathrm{PK}$ model the Uemura-type plots can be obtained, as it was previously deduced for the AH model [13] and most recently also for the extended Hubbard model with intersite attraction [15, 16]. Our results support the conclusion $[15,16]$ that the reason for the Uemura scaling in the short coherence length superconductors including the cuprates, bismuthates, and the organic materials is connected with a separation of the energy scales for pairing and for the phase coherence in the low concentration (underdoped) regime or in the strong coupling.

Comparing the PK model with the attractive Hubbard model one finds in both cases gradual evolution from the BCS-like limit to the tightly bound pairs regime. Also in both models the interaction stabilizes the on-site $s$-wave superconductivity for any coupling strength. Due to the nonlocal pairing mechanism (intersite charge exchange) the dynamics of electron pairs in the PK model is qualitatively different from that in the AH model $[2,10]$. It results in different thermodynamic and electrodynamic properties of both models. In the AH model with increasing $|U|$ the $T_{\mathrm{c}}$ increases exponentially for small $|U|$, then it goes through a round maximum and it decreases as $t^{2} /|U|$ for large coupling $[10,12,13]$. Analogous behavior is also found for the thermodynamic critical field $H_{\mathrm{c}}$ at $T=0$ $[10,13 \mathrm{~b}]$. On the contrary, in the PK model there is no maximum of $T_{\mathrm{c}}$ and $H_{\mathrm{c}}^{2}$ at intermediate $J / t$ and both these quantities increase linearly with $J$ for large coupling (cf. Fig. 4, Appendix B and Refs. [2, 8]). Also the behavior of the penetration depth $\lambda$ and the pair mobility $t_{\mathrm{p}}$ is different (cp. Fig. 1). The pair mobility $t_{\mathrm{p}} \sim 1 / \lambda^{2}$ increases with $J$ in $\mathrm{PK}$ model $\left(1 / \lambda^{2} \sim J\right.$ for $\left.t \rightarrow 0\right)$, while it decreases with $|U|$ in the AH model $\left(1 / \lambda^{2} \sim t^{2} /|U|\right.$ for $\left.t \rightarrow 0\right)$. It is in agreement with studies of collective excitations performed using a generalized random-phase approximation $[19,20]$. The collective mode velocity increases with $J$ in the PK model [19], in contrast to the $\mathrm{AH}$ model where it decreases with the coupling $|U|[20]$. 


\section{Acknowledgments}

We thank B. Bułka, R. Micnas, B. Tobijaszewska, and T. Kostyrko for helpful discussion. This work is supported in part from the State Committee for Scientific Research project no. 2 P03B 03717.

\section{Appendix A}

In the limits of weak and strong coupling the system of self-consistent Eqs. (3) at $T=0$ can be analytically solved and one can derive explicit expressions for various superfluid characteristics.

Below we summarize the asymptotic expressions determining the gap parameter $E_{\mathrm{g}}(0)$ and the superfluid stiffness $\rho_{\mathrm{S}}(0)=\frac{\hbar^{2} e^{2}}{16 \pi e^{2}} \lambda^{-2}(0)$, derived for (a) $n=1$ in the case of $d=2 \mathrm{SQ}$ lattice and for (b) $0<n<2$ in the case of rectangular DOS:

$$
D(E)=\left\{\begin{array}{cc}
\frac{1}{B}, & \text { for }-\frac{B}{2}<E<\frac{B}{2}, \\
0, & \text { otherwise, } \quad B=2 z t .
\end{array}\right.
$$

\section{A.1. The weak-J case}

Case (a)

$$
\begin{aligned}
& E_{\mathrm{g}}(0)=2 \Delta=2 \mathrm{zt} \exp \left(-\pi \sqrt{\frac{t}{J}}\right) \\
& \rho_{\mathrm{s}}(0)=\frac{\hbar^{2} c^{2}}{16 \pi e^{2}} \lambda^{-2}(0)=\frac{2}{\pi^{2}} t+\left(2-\frac{1}{2 \pi}\right) \frac{t^{2}}{J} \exp \left(-2 \pi \sqrt{\frac{t}{J}}\right) .
\end{aligned}
$$

Case (b)

$$
\Delta=\bar{B} \sqrt{n(2-n)} \exp \left(-\frac{\bar{B}}{J_{0}}\right),
$$

$$
\rho_{\mathrm{s}}(0)=\frac{1}{4}|t| n(2-n)\left[1+14 \exp \left(-\frac{2 \bar{B}}{J_{0}}\right)\right]
$$

where $\bar{B} \approx 2 z t\left[1+\frac{1}{8} n(2-n) \frac{J}{t}\right]$.

\section{A.2. The strong-J case}

Case (a)

$$
E_{\mathrm{g}}=2 \Delta=2 J_{0} x_{\mathrm{s}}=4 J\left[1-\left(\frac{2 t}{3 J}\right)^{2}\right]
$$




$$
\rho_{\mathrm{s}}=\frac{1}{2}\left[J-\frac{2 t^{2}}{9 J}-\mathrm{O}\left(\frac{t^{4}}{J^{3}}\right)\right] .
$$

Case (b)

$$
\begin{aligned}
& \Delta=\frac{J_{0}}{2}\left[1-\frac{1}{6}\left(\frac{\bar{B}}{J_{0}}\right)^{2}\right] \sqrt{n(2-n),} \\
& \rho_{\mathrm{S}}(0)=\frac{1}{2} n(2-n) J\left[1-\frac{1}{6}\left(\frac{\bar{B}}{J_{0}}\right)^{2}\right],
\end{aligned}
$$

where $\bar{B} \approx 2 z t\left[1+\frac{1}{6} n(2-n)\right]$.

\section{Appendix B}

The $T_{\mathrm{p}}$ is determined by three self-consistent Eqs. (3) taken in the limit $\Delta \rightarrow 0$, whereas the $T_{\mathrm{c}}$ is given by the set of four equations (8) and (3). Obviously, $T_{\mathrm{c}} \leq T_{\mathrm{p}}$.

In the limits of weak and strong coupling one can derive several analytical expressions for both these quantities. As in Appendix A we will focus on the case of $d=2 \mathrm{SQ}$ lattice and that of rectangular DOS.

\section{B.1. The weak-J case}

Close to $T_{\mathrm{p}}$ Eq. (7) reduces to

$$
\frac{\lambda^{2}(0)}{\lambda^{2}(T)}=\frac{\rho_{\mathrm{s}}(T)}{\rho_{\mathrm{s}}(0)} \simeq 2\left(1-\frac{T}{T_{\mathrm{p}}}\right) .
$$

For $T_{\mathrm{c}} \lesssim T_{\mathrm{p}}$ Eqs. (8) and (10) yield

$$
k_{\mathrm{B}} T_{\mathrm{c}} \simeq \frac{k_{\mathrm{B}} T_{\mathrm{p}}}{1+\frac{k_{\mathrm{B}} T_{\mathrm{p}}}{\pi \rho_{\mathrm{s}}(0)}}
$$

which determines $T_{c}$ in the weak coupling limit and shows that for $J / t \rightarrow 0$ we recover asymptotically the HFA transition temperature $\left(T_{c} / T_{\mathrm{p}} \rightarrow 1\right)$.

Explicit form of $T_{\mathrm{c}}$ (Eq. (10)) can be found taking into account analytical expressions for $T_{\mathrm{P}}$ and $\rho_{\mathrm{s}}(0)$ for $J / t \ll 1$. In the case of SQ lattice for $n=1$ the $T_{\mathrm{p}}$ determined from Eqs. (3) and $\rho_{\mathrm{s}}(0)$ taken from $(10)$ are

$$
\begin{aligned}
& k_{\mathrm{B}} T_{\mathrm{p}} \simeq 2.28 t \exp \left(-\pi \sqrt{\frac{t}{J}}\right), \\
& \rho_{\mathrm{S}}(0) \simeq \frac{2}{\pi^{2}} \mathrm{t}
\end{aligned}
$$


whereas in the case of rectangular DOS and $0<n<2$ one obtains

$$
\begin{aligned}
& k_{\mathrm{B}} T_{\mathrm{p}} \simeq 0.57 \widetilde{B} \sqrt{n(2-n)} \exp \left(-\frac{\widetilde{B}}{J_{0}}\right), \\
& \rho_{\mathrm{s}}(0) \simeq \frac{1}{4}|t| n(2-n),
\end{aligned}
$$

where $\widetilde{B}=2 z t+4 p J, p \simeq \frac{z}{16} n(2-n)$.

\section{B. D. The strong-J case}

As it follows from numerical solutions of Eq. (8) (cf. Fig. 6), for $J / t \gg 1$ the $T_{\mathrm{c}}$ for $d=2$ is well approximated by $\rho_{\mathrm{s}}(0)$, i.e.

$$
k_{\mathrm{B}} T_{\mathrm{c}} \simeq \frac{\pi}{2} \rho_{\mathrm{s}}(0)
$$

Using the results for $\rho_{\mathrm{s}}(0)$ derived in Appendix $\mathrm{A}$ and calculating asymptotic expressions for $T_{\mathrm{p}}$ from Eqs. (3), one obtains:

a) $t=0, \quad 0<n<2$

$$
\begin{aligned}
& k_{\mathrm{B}} T_{\mathrm{p}}=d J \frac{n-1}{\ln \frac{n}{2-n}}, \\
& k_{\mathrm{B}} T_{\mathrm{c}}=\frac{1}{2} \frac{\pi}{2} J n(2-n), \\
& E_{\mathrm{g}}(0)=2 \sqrt{\mu^{2}+\Delta^{2}}=2 d J,
\end{aligned}
$$

b) $t / J \ll 1, n=1$, SQ lattice

$$
\begin{aligned}
& k_{\mathrm{B}} T_{\mathrm{p}} \simeq J\left[1-\left(\frac{2 t}{3 J}\right)^{2}\right], \\
& k_{\mathrm{B}} T_{\mathrm{c}}=\frac{1}{2} \frac{\pi}{2} J\left[1-\frac{2}{9}\left(\frac{t}{J}\right)^{2}\right],
\end{aligned}
$$

c) $t / J \ll 1$, rectangular DOS, $0<n<2$

$$
\begin{aligned}
& k_{\mathrm{B}} T_{\mathrm{p}} \simeq d J\left[1-\frac{2}{3}\left(\frac{t}{J}\right)^{2}\right] \frac{n-1}{\ln \frac{n}{2-n}}, \\
& k_{\mathrm{B}} T_{\mathrm{c}} \simeq \frac{1}{2} \frac{\pi}{2} n(2-n) J\left[1-\frac{2}{3}\left(\frac{t}{J}\right)^{2}\right] .
\end{aligned}
$$

As we can see, in the strong coupling limit the ratio $T_{\mathrm{c}} / T_{\mathrm{p}}$ substantially depends on the band filling. It is maximal for $n=1$ 


$$
\frac{T_{\mathrm{c}}}{T_{\mathrm{p}}}(n=1) \Rightarrow \frac{\pi}{4}
$$

and decreases towards zero with the increase in $|n-1|$ :

$$
\frac{T_{\mathrm{c}}}{T_{\mathrm{p}}}(n) \simeq \frac{\left(\ln \frac{n}{2-n}\right) n(2-n)}{n-1} \frac{\pi}{8} .
$$

\section{References}

[1] K.A. Penson, M. Kolb, Phys. Rev. B 33, 1663 (1986).

[2] S. Robaszkiewicz, B. Bułka, Phys. Rev. B 59, 6430 (1999) and references therein.

[3] I. Affleck, J.B. Marston, J. Phys. C 21, 2511 (1988).

[4] A.E. Sikkema, I. Affleck, Phys. Rev. B 52, 10207 (1995).

[5] M. van den Bossche, M. Caffarel, Phys. Rev. B 54, 17414 (1996).

[6] G. Bouzerar, G.I. Japaridze, Z. Phys. B 104, 215 (1997).

[7] G.I. Japaridze, E. Muller-Hartmann, J. Phys., Condens. Matter 9, 10509 (1997).

[8] W.R. Czart, S. Robaszkiewicz, Acta Phys. Pol. A 97, 217 (2000).

[9] D.J. Scalapino, S.R. White, S. Zhang, Phys. Rev. B 47, 7995 (1993).

[10] (a) W.R. Czart, T. Kostyrko, S. Robaszkiewicz, Physica C 272, 51 (1996); (b) B. Bułka, S. Robaszkiewicz, Phys. Rev. B 54, 13138 (1996).

[11] J.M. Kosterlitz, D.J. Thouless, J. Phys. C 6, 1181 (1973).

[12] P.J.H. Denteneer, Guozhong An, M.J. van Leeuven, Phys. Rev. B 47, 6256 (1993); M.J. van Leeuven, M.S.L. du Crao de Jongh, P.J.H. Denteneer, J. Phys. A 29, 41 (1996).

[13] (a) J.M. Singer, T. Schneider, M. Pedersen, Eur. Phys. J. B 2, 17 (1998); (b) M. Bąk, R. Micnas, J. Phys., Condens. Matter 10, 9029 (1998).

[14] B. Chattopadhyay, Phys. Lett. A 226, 231 (1997); B. Chattopadhyay, D.M. Gaitonde, A. Taraphder, Europhys. Lett. 34, 705 (1996).

[15] R. Micnas, S. Robaszkiewicz, B. Tobijaszewska, J. Supercond. 12, 79 (1999).

[16] B. Tobijaszewska, R. Micnas, Acta Phys. Pol. A 97, 393 (2000); R. Micnas, B. Tobijaszewska, unpublished.

[17] Y.J. Uemura, G.M. Luke, B.J. Sternlieb, J.H. Brewer, J.F. Carolan, W.N. Hardy, R. Kadono, J.R. Kempton, R.F. Kiefl, S.R. Kreitzman, P. Mulhern, T.M. Riseman, D. Ll. Williams, B.X. Yang, S. Uchida, H. Takagi, J. Gopalakrishnan, A.W. Sleight, M.A. Subramanian, C.L. Chien, M.Z. Cieplak, Gang Xiao, V.Y. Lee, B.W. Statt, C.E. Stronach, W.J. Kossler, X.H. Yu, Phys. Rev. Lett. 62, 2317 (1989); ibid. 66, 2665 (1991).

[18] Y.J. Uemura, Physica C 282-287, 194 (1997).

[19] G.K. Roy, B. Bhattacharyya, Phys. Rev. B 55, 15506 (1997).

[20] T. Kostyrko, R. Micnas, Phys. Rev. B 46, 11025 (1992). 